\title{
Nonthermal breaking of magnetic order via photogenerated spin defects in the spin-orbit coupled insulator $\mathrm{Sr}_{3} \mathrm{Ir}_{2} \mathrm{O}_{7}$
}

\author{
Ernest Pastor $\odot,{ }^{1, *}, \dagger$ David Moreno-Mencía $\odot,{ }^{1, *}$ Maurizio Monti $\odot,{ }^{2}$ Allan S. Johnson $\odot,{ }^{1}$ Nina Fleischmann,${ }^{1,2}$ \\ Cuixiang Wang, ${ }^{3}$ Youguo Shi, ${ }^{3}$ Xuerong Liu, ${ }^{4}$ Daniel G. Mazzone,${ }^{5}$ Mark P. M. Dean $\odot,{ }^{6}$ and Simon Wall $\odot, 2, \$$ \\ ${ }^{1}$ ICFO-Institut de Ciències Fotòniques, The Barcelona Institute of Science and Technology, 08860 Castelldefels, Barcelona, Spain \\ ${ }^{2}$ Department of Physics and Astronomy, Aarhus University, Ny Munkegade 120, 8000 Aarhus C, Denmark \\ ${ }^{3}$ Beijing National Laboratory for Condensed Matter Physics, Institute of Physics, Chinese Academy of Sciences, Beijing 100190, China \\ ${ }^{4}$ School of Physical Science and Technology, ShanghaiTech University, Shanghai 201210, China \\ ${ }^{5}$ Laboratory for Neutron Scattering and Imaging, Paul Scherrer Institut, CH-5232 Villigen, Switzerland \\ ${ }^{6}$ Condensed Matter Physics and Materials Science Department, Brookhaven National Laboratory, Upton, New York 11973, USA
}

(Received 2 November 2021; revised 22 December 2021; accepted 25 January 2022; published 7 February 2022)

\begin{abstract}
In many strongly correlated insulators, antiferromagnetic order competes with exotic and technologically relevant phases, like superconductivity. While control of spin order is critical to stabilize different functional states, elucidating the mechanism of laser-induced demagnetization in complex oxides remains a challenge. It is unknown if the optical pulse can quench magnetization nonthermally or if it instead only acts as a heat source. Here, we use ultrafast, broadband, optical spectroscopy to track the responses of the electronic, lattice, and spin degrees of freedom and their relation to antiferromagnetism in the strongly spin-orbit coupled insulator $\mathrm{Sr}_{3} \mathrm{Ir}_{2} \mathrm{O}_{7}$. We find that magnetization can be rapidly and strongly suppressed on a sub-150 fs timescale. At low excitation fluences, the magnetic recovery is fast; however, the recovery time increases dramatically with the magnitude of demagnetization. At the same time, we show that the lattice, evidenced through the $A_{g}$ phonon frequencies, appears to remain below $T_{\mathrm{N}}$, suggesting that the system remains nonthermal during the optical modulation of spin order. We suggest that photogenerated spin defects are responsible for the long-lived demagnetized state and discuss its implications for optical control of solids.
\end{abstract}

DOI: 10.1103/PhysRevB.105.064409

\section{INTRODUCTION}

In Mott insulators, the breakdown of long-range antiferromagnetic (AFM) spin order into phases such as high-temperature superconductivity through doping is a key method to control quantum materials in equilibrium [1,2]. Out of equilibrium, femtosecond light pulses have emerged as a powerful tool to access nonequilibrium phases, but the role played by the spin degree of freedom remains poorly understood. It remains unknown if optical quenching of spin order requires a concomitant heating of the lattice, i.e., if the suppression of spin order is due to rapid but trivial heating or if, in contrast, the suppression is nonthermal, potentially enabling access to transient states unreachable in thermodynamic equilibrium [3]. Central to understanding this issue is understanding how photoexcitation influences all degrees of freedom in the system. However, tracking many different degrees of freedom often requires the use of multiple techniques, sometimes operating in very different regimes [4], and such an approach can lead to artifacts [5].

Ultrafast optical spectroscopy is a powerful method for tracking structural and electronic transitions. Structural

\footnotetext{
*These authors contributed equally to this work.

†ernest.pastor@icfo.eu

†simon.wall@phys.au.dk
}

changes can be inferred from the dynamics of the coherent phonon response [6,7], while electronic changes can be modeled through changes in the optical conductivity [8]. However, finding unique optical markers for antiferromagnetism is particularly challenging, as the lack of net magnetic moment hinders the use of traditional magneto-optical methodologies. Such difficulty has stimulated the development of methods to probe it on fast timescales, but many require access to large-scale user facilities, which makes collecting full datasets difficult, and often, sensitivity to magnetic order comes at the expense of sensitivity to other degrees of freedom [2,9-16].

Here, we demonstrate that broadband four-dimensional (4D) optical spectroscopy can extract quantitative information on the electronic, structural, and magnetic degrees of freedom in an AFM insulator. We record the transient optical response of a correlated AFM insulator in $4 \mathrm{D}$, namely, as a function of time, wavelength, excitation fluence, and temperature. This multidimensional approach allows us to separate out magnetic dynamics from structural and electronic processes, enabling us to map out quantitative magnetic changes while also assessing the lattice temperature from the viewpoint of the phonon frequencies. We show that, in this system, moderate photoexcitations cause a large $\sim 50 \%$ and prompt suppression of magnetic order which can recover rapidly (under a picosecond). In contrast, at higher excitation densities, magnetic order can be completely suppressed, resulting in a dramatic increase of the recovery time. Crucially, we demonstrate that the lattice 
temperature remains below the equilibrium Néel temperature $\left(T_{\mathrm{N}}\right)$, while the system is in the demagnetized state, showing that the long-lived state is nonthermal. Our experiments and simulations suggest that the formation of photo-induced spin defects dictates the stability of the demagnetized state and the recovery of magnetic order. The picture that emerges from our data is one in which the degree of disorder of the system plays a dominant role in determining the fate of the photoexcited state, offering an opportunity to tune AFM order without changing the temperature.

We focus on the Ruddlesden-Popper family of iridium oxides with the formula $\mathrm{Sr}_{n+1} \mathrm{Ir}_{n} \mathrm{O}_{3 \mathrm{n}+1}$ in which $n$ indicates the number of $\mathrm{SrIrO}_{3}$ perovskite layers located in between $\mathrm{SrO}$ layers $[17,18]$. These oxides have raised much interest due to their similarities with superconducting cuprates and because their strong spin-orbit coupling (SOC) generates a $J_{\text {eff }}=\frac{1}{2}$ insulating state [Fig. 1(b)] that can lead to additional functionality [19-21]. Herein, we evaluate the bilayer $(n=2)$ system $\mathrm{Sr}_{3} \mathrm{Ir}_{2} \mathrm{O}_{7}$, which displays an out-of-plane collinear AFM insulating state that emerges from a paramagnetic (PM) insulating state at the Néel temperature $T_{\mathrm{N}} \sim 280 \mathrm{~K}$ [22]. Unlike $\mathrm{Sr}_{2} \mathrm{IrO}_{4}$ $(n=1)$, the spin-wave dispersion in $\mathrm{Sr}_{3} \mathrm{Ir}_{2} \mathrm{O}_{7}$ is gapped. As a result, high-energy modes cannot decay through coupling to lower energy modes, and decay can only occur through coupling to other degrees of freedom. This can slow thermalization of the spin system with other degrees of freedom and may enable long-lived nonthermal states. Indeed, timeresolved resonant inelastic x-ray scattering measurements of $\mathrm{Sr}_{3} \mathrm{Ir}_{2} \mathrm{O}_{7}$ have shown spin excitations throughout the magnetic Brillouin zone [20] which are longer lived than their counterparts in $\mathrm{Sr}_{2} \mathrm{IrO}_{4}$ [9]. However, the nonthermal nature of the transient state could not be verified. In the following, we show how broadband optical spectroscopy can complete this goal and shed light onto the demagnetization mechanism.

Our paper is structured as follows: in Sec. II, we discuss the origin of the static optical properties of $\mathrm{Sr}_{3} \mathrm{Ir}_{2} \mathrm{O}_{7}$ as well as how the optical region is sensitive to magnetic order and how photoexcitation changes the optical spectra both above and below the Néel temperature. In Sec. III, we show how, through wavelength selectivity, we can isolate the magnetic dynamics from the other degrees of freedom, enabling us to extract detailed information on the magnetic response as a function of fluence, time, and temperature. In Sec. IV, we then focus on the response of the lattice obtained from the coherent phonon response and show that, despite a significant thermal redshift in the phonon mode frequency with temperature, no redshift is observed after photoexcitation, indicating that the lattice is in a nonthermal state. In addition, we discuss how the phonons also influence the electronic properties of the material. Finally, in Sec. V, we present a discussion of thermal and nonthermal scenarios for $\mathrm{Sr}_{3} \mathrm{Ir}_{2} \mathrm{O}_{7}$ and present simple Monte Carlo simulation to demonstrate how increasing light-induced disorder can explain the spin dynamics without need for a temperature change.

\section{TEMPERATURE AND LIGHT-INDUCED CHANGES IN REFLECTIVITY}

Details of the sample can be found in the Supplemental Material [23] (see also Ref. [24] therein). We start by evalu-

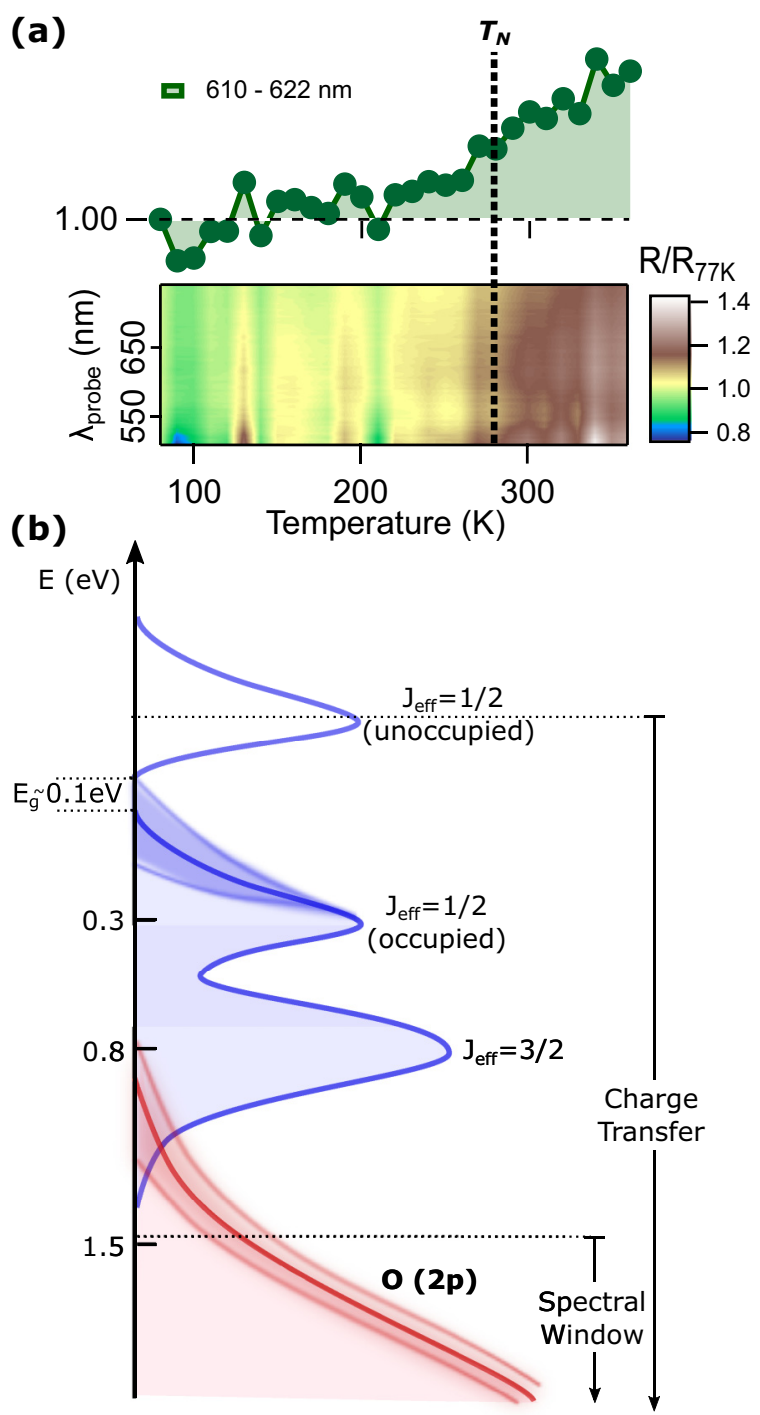

FIG. 1. $\mathrm{Sr}_{3} \mathrm{Ir}_{2} \mathrm{O}_{7}$ and its optical response. (a) Temperature dependence of the static reflectivity above and below the Néel temperature $T_{\mathrm{N}} \sim 280 \mathrm{~K}$. The signal is shown relative to the value at $77 \mathrm{~K}$ [antiferromagnetic (AFM)]. Top: Lineout at $\sim 610 \mathrm{~nm}$ showing a change in reflectivity between low and high temperatures. For clarity, the signal is smoothed; raw data are displayed in Fig. S1 in the Supplemental Material [23]. (b) Schematic summary of the electronic structure of $\mathrm{Sr}_{3} \mathrm{Ir}_{2} \mathrm{O}_{7}$ [25]. The strong spin-orbit coupling generates a $J_{\text {eff }}=\frac{1}{2}$ Mott-like insulating state characterized by relatively small bandgap $\left(E_{g}\right)$, a filled lower Hubbard band (LHB) and an empty upper Hubbard band (UHB). The spectral window of our broadband experiments is primarily sensitive to changes in the amplitude and energy of the charge transfer resonance induced by the magnetic, structural, and electronic degrees of freedom.

ating the change in reflectivity as a function of temperature [Fig. 1(a)]. We focus on the visible region which, as depicted in Fig. 1(b), primarily probes charge transfer transitions involving lower-lying $\mathrm{O} 2 p$ and $\operatorname{Ir} 5 d$ states to unoccupied states near the bandgap. As the bandgap has a Slater-like character, it is thus expected to be magnetically sensitive $[17,25,26]$, with long-range magnetic order perturbing the optically probed magnetic structure. Indeed, we observe that 

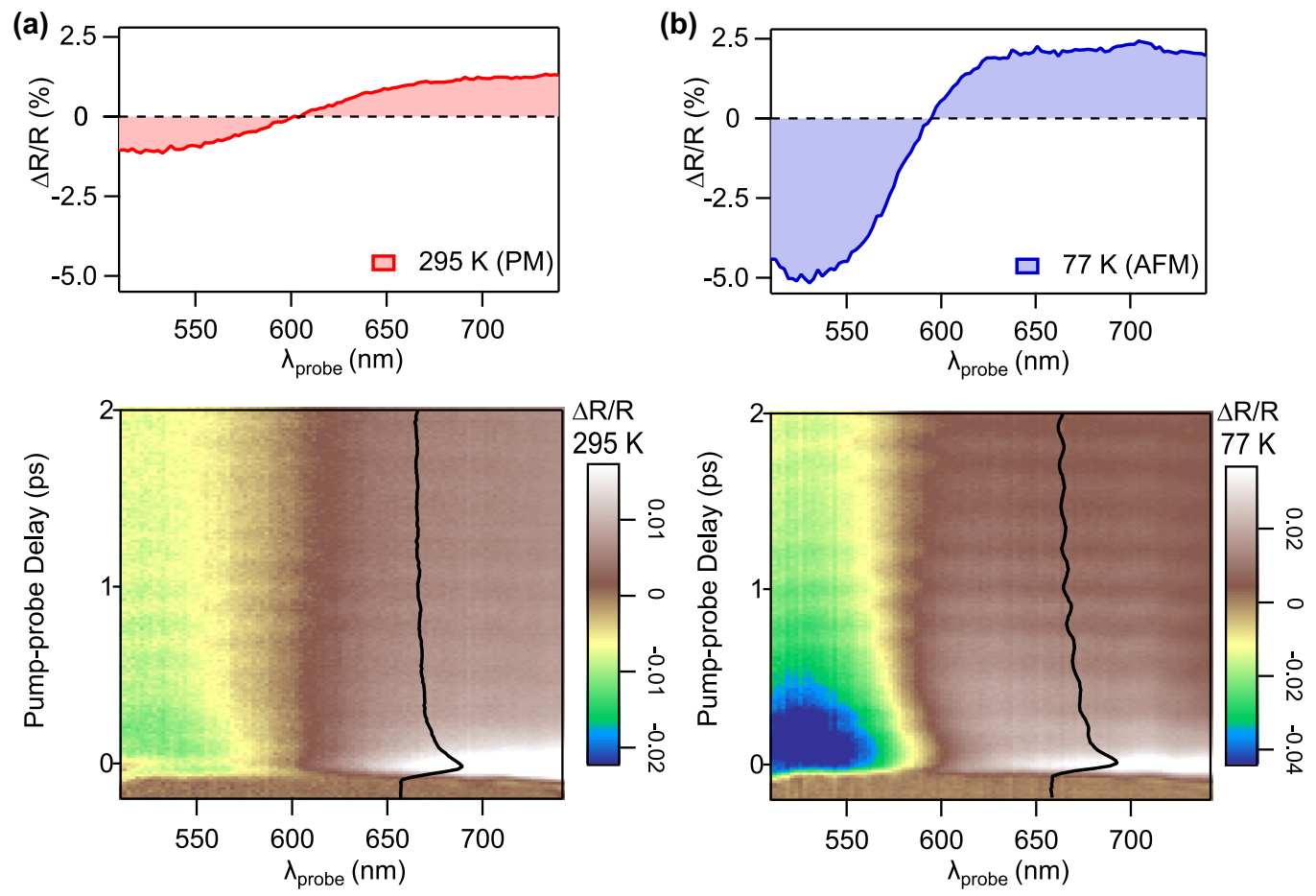

FIG. 2. Transient reflectivity response. Transient reflectivity change upon $800 \mathrm{~nm}$ excitation $\left(F=5 \mathrm{~mJ} \mathrm{~cm}^{-2}\right)$ at (a) $295 \mathrm{~K}$ [paramagnetic (PM)] and (b) $77 \mathrm{~K}$ [antiferromagnetic (AFM)]. For each temperature, the top panel shows a lineout at 0.14 ps time delay. Also displayed is an example of the coherent oscillations that modulate the transient response (obtained within 650-700 nm range). The amplitude of the oscillations is notably larger at $77 \mathrm{~K}$ when the sample is in the AFM state (for full kinetic traces see the Supplemental Material [23]).

the static reflectivity significantly increases above $T_{\mathrm{N}}$, but the change is gradual and not abrupt at the transition temperature [Fig. 1(a)]. This likely reflects the fact that the optical changes are not exclusively associated with magnetism but also depend on the electronic and structural degrees of freedom. As these also evolve with temperature, isolating the magnetic signal is nontrivial and hinders accurate quantification of magnetic order from the reflectivity alone.

This challenge can, however, be overcome with ultrafast broadband spectroscopy. Figure 2(a) shows typical broadband transient reflectivity spectra taken above and below $T_{\mathrm{N}}$ after photoexcitation with $800 \mathrm{~nm}\left(1.5 \mathrm{eV}, F=5 \mathrm{~mJ} \mathrm{~cm}^{-2}\right)$ laser light (other fluences displayed in Fig. S2 in the Supplemental Material [23]). The spectral changes are qualitatively similar in both cases. Both temperatures show a suppressed reflectivity at short wavelengths and an increase $>\sim 600 \mathrm{~nm}$ (see lineouts on the top panel) consistent with a photo-induced shift in the O $2 p$-Ir $5 d$ charge transfer resonance [red line in Fig. 1(b)], like what is seen in the cuprates [27]. Moreover, both temperatures exhibit fast dynamics within the first picosecond, followed by a much slower plateau. The signal is also modulated by an oscillating component due to coherent phonons, which will be discussed in Sec. IV.

At room temperature, where the system has no magnetic order, the dynamics result from purely electronic and structural changes. Below $T_{\mathrm{N}}$, the spectral response is similar but with a different magnitude. We observe a significant signal enhancement $\sim 500-650 \mathrm{~nm}$, while the changes $>650 \mathrm{~nm}$ are less abrupt. In addition, the kinetic lineouts show a marked increase in the amplitude of the oscillations with decreasing temperature, as discussed later. While the different response between the AFM and PM states indicate a fingerprint of magnetic behavior, it remains unclear whether the observed optical signals can be attributed only to the magnetic degrees of freedom.

\section{OPTICAL FINGERPRINTS OF AFM ORDER}

In general, each degree of freedom will produce a different spectral change in the dielectric function, which is the property measured in our experiment. In the linear approximation, one can then express the measured change in the reflectivity due to the changing dielectric function as

$$
\delta R=\frac{d R}{d \epsilon}\left(\frac{d \epsilon}{d C} \delta C+\frac{d \epsilon}{d Q} \delta Q+\frac{d \epsilon}{d M} \delta M\right),
$$

where $C, Q$, and $M$ represent the charge, structural, and magnetic degrees of freedom, respectively. These three terms will have different wavelength dependences; therefore, to establish which optical region has the most sensitivity to magnetic order and least sensitivity to the other degrees of freedom, we measure a 4D dataset, recording the response of the system as a function of probe wavelength $\lambda$, fluence $F$, temperature $T$, and time $t$. Our aim is to identify a region of the spectra that is most insensitive to thermal changes in the electronic and structural properties above $T_{\mathrm{N}}$. This allows us to subsequently subtract these contributions from the signal below $T_{\mathrm{N}}$ and thus to isolate the magnetic response. Figure 3(a) shows a detailed temperature scan at different probe wavelengths for a fixed fluence and delay, which exemplifies the merits of our approach. From the data in Fig. 2 alone (comparison of spectra at 295 and $77 \mathrm{~K}$ ), one might assume that the 500-550 nm region would exhibit the largest magnetic 

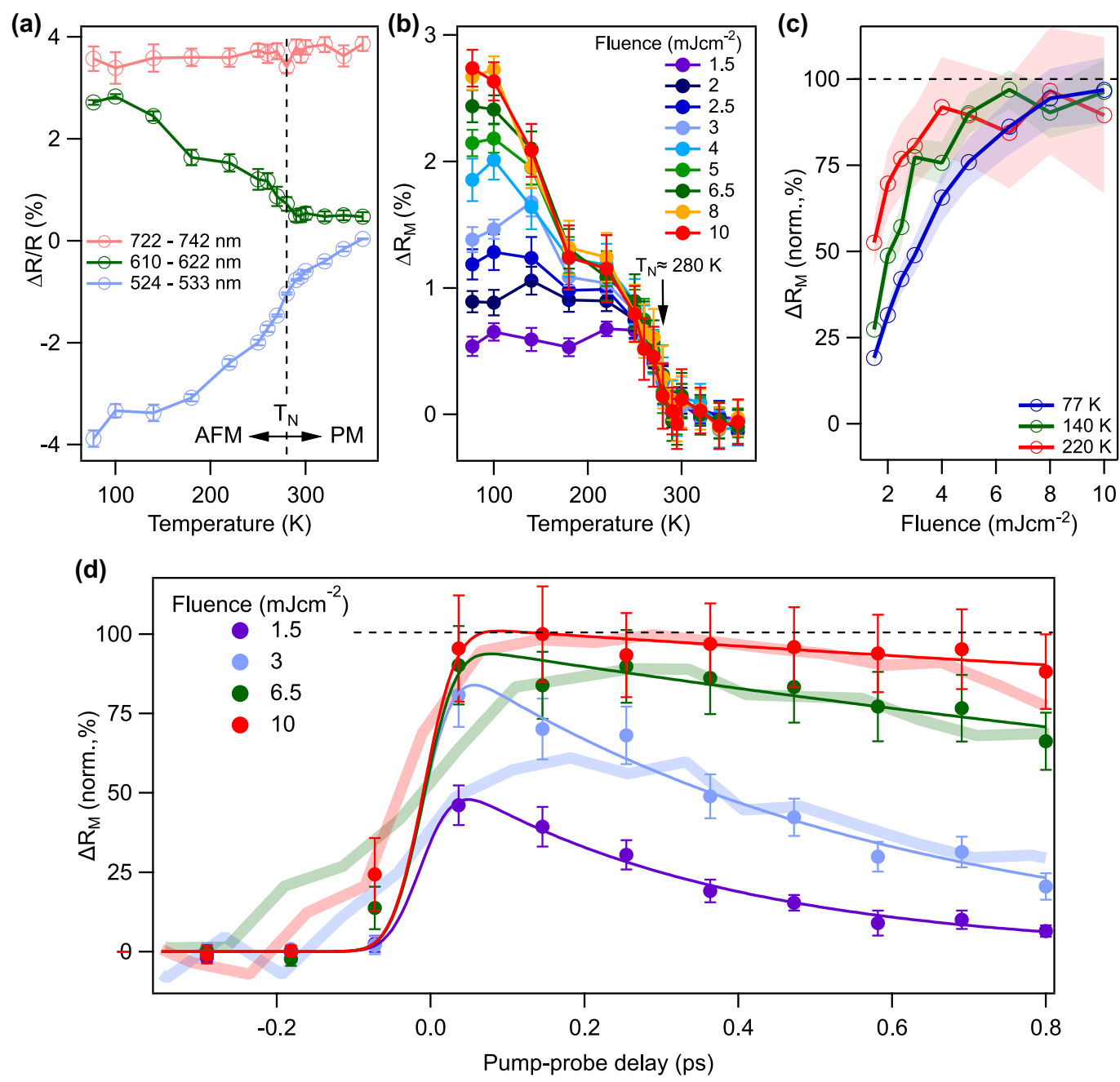

FIG. 3. Changes in the magnetic degree of freedom. (a) Temperature dependence of the transient reflectivity at different probe wavelengths. For clarity, the traces are offset. The $610 \mathrm{~nm}$ region is both sensitive to magnetic order while showing a temperature-independent response above $T_{\mathrm{N}}$. (b) Temperature dependence of the magnetic degree of freedom $\left(\Delta R_{M}\right)$ at different excitation fluences measured at $360 \mathrm{fs}$ time delay obtained as $\Delta R(T)-\Delta R\left(T>T_{\mathrm{N}}\right)$ in the $610 \mathrm{~nm}$ region. The signal saturates at high fluences and at temperatures close the Néel temperature, as expected for light-induced demagnetization. We normalize the maximum $\Delta R_{M}$ value at $10 \mathrm{~mJ} \mathrm{~cm}^{-2}$ to $100 \%$ demagnetization. (c) Fluence dependence of the magnetic signal at different temperatures showing that full demagnetization is more rapidly attained at higher temperatures. (d) Time evolution of the magnetic signal measured at $77 \mathrm{~K}$ at different fluences showing fast and large (>40\%) demagnetization as well a change from rapid recovery to permanent demagnetization at $F>4 \mathrm{~mJ} \mathrm{~cm}^{-2}$ (see also Fig. S5 in the Supplemental Material [23]). For comparison, the time evolution of the magnetic Bragg peak obtained from Ref. [20] is also shown (solid translucent lines, scaled).

sensitivity, as it shows the largest change when crossing $T_{\mathrm{N}}$. However, the full temperature scan reveals that this region is strongly temperature dependent above $T_{\mathrm{N}}$, which precludes extraction of the magnetic signal without a detailed understanding of the nonmagnetic temperature evolution of the system. Likewise, at long wavelengths $(>700 \mathrm{~nm})$ the signal shows a temperature-independent response above $T_{\mathrm{N}}$ but lacks magnetic sensitivity below $T_{\mathrm{N}}$. Between these two extremes, we find that wavelengths $\sim 610 \mathrm{~nm}$ are unique because the response is temperature independent above $T_{\mathrm{N}}$ while retaining sensitivity to magnetic order. This remains true for all probed fluences and time delays, as shown in Fig. S3 in the Supplemental Material [23] and as discussed below.

We explore the feasibility of isolating the magnetic component by subtracting the electronic temperatureindependent contribution from the total reflectivity,
$\Delta R(T)-\Delta R\left(T>T_{\mathrm{N}}\right)$ centered at $610 \mathrm{~nm}$. We label this assay of magnetism as $\Delta R_{M}$. Figure 3(b) shows this analysis for data collected at $360 \mathrm{fs}$ time delay at different excitation conditions (see Fig. S4 in the Supplemental Material [23] for other time delays and details). Above $T_{\mathrm{N}}$, the signal is zero at all fluences, as expected by our methodology. At $T_{\mathrm{N}}$, there is a discontinuity, and the magnetic response rises sharply. Such a jump with temperature contrasts with the much slower change seen in reflectivity measured statically [Fig. 1(a)] and is indicative that our magnetic assay can isolate the light-induced change in magnetic order from the other degrees of freedom. Further corroboration of the magnetic sensitivity can be inferred from the fluence dependence of the signal. Increasing the pump fluence increases the signal, indicating that the system shows a greater degree of demagnetization. Moreover, for the highest fluences, 
the signal saturates, as would be expected for a complete demagnetization [Fig. 3(c)]. Importantly, both the saturation fluence and signal magnitude are temperature dependent. The magnitude of the signal at saturation increases as the sample is cooled below $T_{\mathrm{N}}$; this is indicative of the fact that a greater fraction of the material displays long-range magnetic order at lower temperatures, which can then be melted by the pump pulse and manifest in the optical response. Furthermore, the shift in the saturation fluence with temperature is consistent with the fact that more energy is needed to demagnetize more of the sample. Based on this, we assign the saturation fluence as the fluence required to completely suppress magnetic order. For all temperatures, we observe that fluences $>\sim 4-5 \mathrm{~mJ} \mathrm{~cm}^{-2}$ result in saturation. Thus, we normalize $\Delta R_{M}$ to the value obtained at the highest excitation fluence $\left(F=10 \mathrm{~mJ} \mathrm{~cm}^{-2}\right)$, which provides a scale for a quantitative analysis of the demagnetization. Figure 3(d) shows the change in the normalized magnetic signal at 77 $\mathrm{K}$ when the sample is in the AFM state (see also Fig. S5 in the Supplemental Material [23]). We observe a sharp change in the magnetic signal immediately after photoexcitation. Notably, we find a very large ( $>50 \%$ ) demagnetization even at low fluences, which is a strong indicator that magnetic order can be efficiently perturbed by the laser pulse in this material.

We note that our conclusions rely on two assumptions. Firstly, we assume that higher-order cross-couplings between the different degrees of freedom, not considered in Eq. (1), do not significantly impact the signal. Second, we assume that the high-temperature response remains constant below $T_{\mathrm{N}}$. Neither case is expected to strictly hold. For example, the phonons dephase less at lower temperatures. Thus, subtracting the high-temperature phonon response from the low-temperature data will still show a residual coherent oscillation that should not be attributed to a modulation on the magnetic response. However, we assume that these deviations and others are small compared with the resolution of our data.

To confirm the validity of our approach, we overlay our signal with the time evolution of a magnetic Bragg peak measured at several (high) fluences using $\mathrm{x}$-ray free-electron laser (XFEL) radiation, which is a direct probe of magnetic order [20]. Due to difference in the pump wavelength and differences in penetration depth of the probe, the two datasets need to be scaled in fluence (see the Supplemental Material [23]), but the agreement between the optical and x-ray data is remarkable and demonstrates that the potential issues with our method of analysis do not have a quantitative influence on our results within our measurement accuracy. The identification of a reliable marker of magnetic order via reflectivity measurements, while not a direct probe of the spin system, provides a tool to systematically map out the response of the sample under a broad range of experimental conditions to those achievable during XFEL measurement.

Inspection of the data in Fig. 3(d) reveals that the recovery dynamics of the spin system are strongly fluence dependent. After the initial rapid demagnetization step, at low fluences, the signal decays and recovers rapidly. In contrast, as saturation fluence is reached, only a minimal decay within $1 \mathrm{ps}$ occurs, indicating near-complete and long-lasting demagnetization. The observed fluence dependence suggests that the laser excitation can trigger different mechanisms of demagnetization and remagnetization depending on the strength of the perturbation. This behavior can have important implications for optical control of magnetism. However, from these data alone, it is challenging to understand what is driving this change in behavior, and measurements of the other degrees of freedom are needed. However, comparing different experimental techniques can be challenging. This is particularly true when comparing excitation fluences often recorded in very different conditions [5]. By validating our optical probe of magnetism, we are now able to confidently compare the response of the charge and structural degrees of freedom with the known suppression of magnetic order.

We wish to understand whether the response of the magnetic system can be simply interpreted in terms of a threetemperature model or whether other mechanisms are at play. In a classical thermal scenario, the photoexcited electrons rapidly heat the spin system above $T_{\mathrm{N}}$, triggering the loss of long-range order. Subsequently, the spins cool by transferring energy to the lattice. After spin-lattice thermalization, if the lattice temperature remains below $T_{\mathrm{N}}$, magnetic order should recover. In contrast, if the lattice temperature rises above $T_{\mathrm{N}}$, the system remains in a demagnetized state until the lattice temperature cools via thermal diffusion, which is generally slow. This behavior has been previously observed in metallic systems [3] and points toward strategies of optical control in which the laser acts effectively as a heat source but may not be applicable in systems with energy gaps that can prevent thermalization. Alternatively, the magnetization dynamics may be nonthermal and follow a different path in which the electronic, spin, and lattice degrees of freedom are not in thermal equilibrium, thus providing exotic paths for optical control. To understand which model applies, it is essential to establish the response of the lattice, and importantly, with optical spectroscopy, these details can be obtained in the same experimental conditions.

\section{TRACKING THE LATTICE DEGREE OF FREEDOM}

To explore the role of the lattice during the magnetic dynamics, we take advantage of the coherent oscillations that modulate the transient reflectivity. Analysis of the high time-resolution scans were performed at room temperature and at $77 \mathrm{~K}$ and under low fluence excitation, which yielded two frequencies at $\sim 146 \mathrm{~cm}^{-1}(4.4 \mathrm{THz})$ and $\sim 182 \mathrm{~cm}^{-1}$ (5.4 THz) corresponding to $A_{1 g}$ modes (see Fig. 4(a) and the Supplemental Material for details [23] and Ref. [28] therein). As expected, these modes redshift at higher temperature with a concomitant decrease in amplitude. The values obtained are in good agreement with those measured with equilibrium Raman spectroscopy [22]. We can then use this temperature dependence of the mode as a proxy for the lattice temperature as we increase the excitation fluence. Figure 4(b) shows that the frequency of both $A_{1 g}$ modes only exhibit a small redshift with fluence, which is less than that of the room temperature value. This strongly points to the fact that, even at high fluences, where magnetism is completely suppressed for multiple picoseconds [20], the lattice heats but remains below $T_{\mathrm{N}}$. 

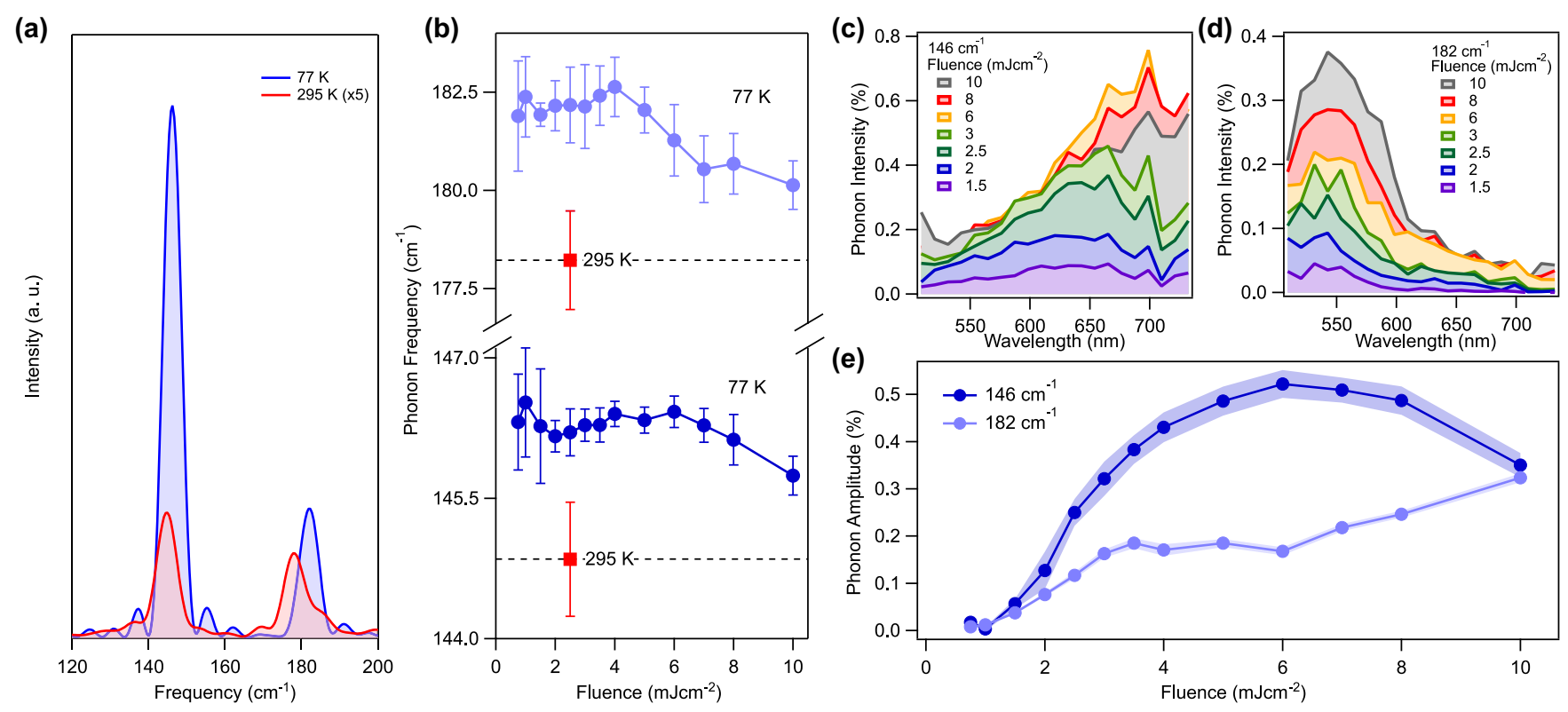

FIG. 4. Changes in the lattice degree of freedom. (a) Fourier transform of the oscillations in the transient reflectivity at $77 \mathrm{~K}$ [antiferromagnetic (AFM)] and $295 \mathrm{~K}$ [paramagnetic (PM)] at $F=2.5 \mathrm{~mJ} \mathrm{~cm}^{-2}$. The characteristic high and low energy $A_{1 \mathrm{~g}}$ modes can be captured. The amplitude decreases and the central frequency shifts with increasing temperature. (b) Change in the mode frequency as a function of excitation fluence at $77 \mathrm{~K}$ (AFM). For comparison, the frequency measured at $295 \mathrm{~K}$ (PM) at low fluences is also displayed (red square). Despite large demagnetization achieved at all fluences, the modes remain mostly unchanged and exhibit only an inflection at the highest fluences. Spectral response of the $146 \mathrm{~cm}^{-1}$ (c) and $182 \mathrm{~cm}^{-1}$ (d) modes as a function of fluence. The $182 \mathrm{~cm}^{-1}$ mode couples to low wavelengths, while the $146 \mathrm{~cm}^{-1}$ mode couples to longer wavelengths and exhibits a redshift with increasing fluence. (e) Maximum amplitude of the phonon modes as a function of fluence (obtained by fitting the spectral response, see the Supplemental Material for details [23]).

Further evidence for a nonthermal state of the system can be found by looking at how the phonon dynamics influence the electronic states. Figures 4(c) and 4(d) show the wavelength dependence of the amplitude of each phonon mode at $77 \mathrm{~K}$ at different fluences. We observe that the $182 \mathrm{~cm}^{-1}$ mode couples almost exclusively to short wavelengths, whereas the $146 \mathrm{~cm}^{-1}$ mode is broader and primarily couples to longer wavelengths. Most strikingly, we find that the peak of the electronic resonance of the $146 \mathrm{~cm}^{-1}$ mode redshifts with increasing excitation, while the other mode remains unchanged. Beyond these spectral changes, we find that the amplitude of the high-frequency mode increases slowly in a linearlike fashion with excitation fluence. In contrast, the low-frequency mode increases linearly and rapidly up to $\sim 4-5 \mathrm{~mJ} \mathrm{~cm}^{-2}$ and subsequently plateaus and exhibits a slight decrease at the highest fluences [Fig. 4(e)]. The redshift in the wavelength maxima is an indicator that the $146 \mathrm{~cm}^{-1}$ mode shifts the charge transfer resonance that dominates the spectral window, as previously observed for the analogous mode in bilayer cuprates [27]. The mode amplitude saturation at moderate fluences is in good agreement with the saturation of the magnetic signal observed in Fig. 3(b) and may suggest a strong link of the $146 \mathrm{~cm}^{-1}$ mode with magnetic order of the system. We note that the fluence at which the amplitude of the mode decreases matches the fluence at which the frequency of the mode starts to shift; this could be the result of more significant lattice heating in the high-photoexcitation regime. Additional corroboration of the sensitivity of the modes to magnetic order can be found in their spectral response at different temperatures. We observe that, above $T_{\mathrm{N}}$, at $295 \mathrm{~K}$, the spectral dependence of the $\sim 182 \mathrm{~cm}^{-1}$ vibration is the same as at
$77 \mathrm{~K}$, while the $\sim 146 \mathrm{~cm}^{-1}$ mode becomes broad and featureless (Fig. S6 in the Supplemental Material [23]), further indicating its high sensitivity to magnetization. However, from optical measurements alone, we cannot determine if the amplitude of the atomic displacements is saturating or if it the coupling of the mode to the electronic states is changing.

\section{DISCUSSION}

We have used broadband optical spectroscopy to identify markers for both the spin and lattice degree of freedom. In agreement with XFEL measurements, we observe that laser excitation can induce large $(>50 \%)$ changes in AFM order even at low fluencies. However, the magnetization dynamics are strongly fluence dependent. Below $\sim 4-5 \mathrm{~mJ} \mathrm{~cm}^{-2}$, the system is rapidly demagnetized and quickly restored. In contrast, at higher fluences, the demagnetization saturates and becomes longer lived. Moreover, by tracking the coherent phonons in the system, we observe that the low-frequency $A_{1 g}$ mode frequency is only moderately perturbed and remains consistent with a low-temperature lattice, despite the complete melting of magnetic order. In addition, the coupling between the phonon mode and the electronic structure also reports evidence of the demagnetized state.

These results strongly suggest that the magnetic system shows a nonthermal response, and the long-lived demagnetized state is not simply a state in which the lattice temperature has risen above $T_{\mathrm{N}}$. Hence, our data rule out multitemperature models [3] and indicate that lattice temperature increases are not required to either suppress long-range spin order or prevent its recovery. Instead, our observations can be explained 
(a)

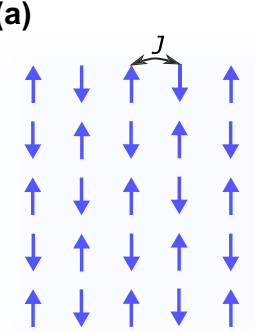

(b)

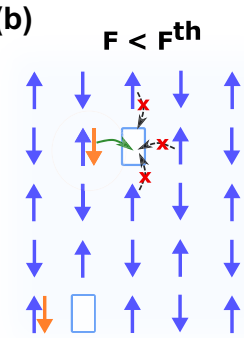

(c)

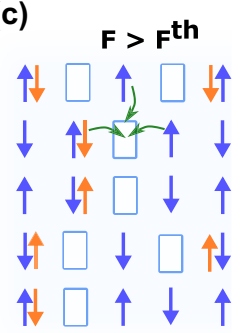

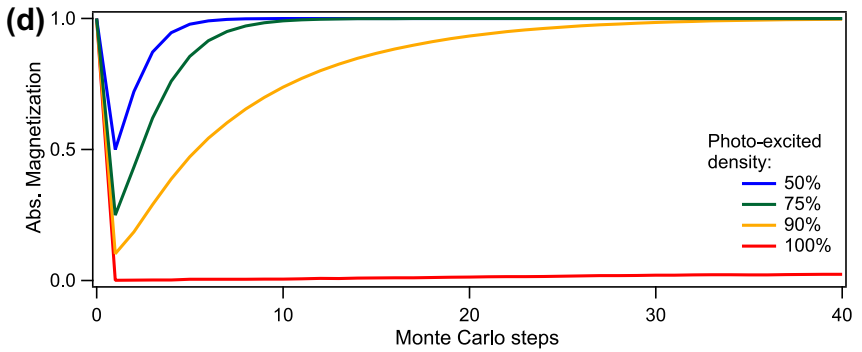

FIG. 5. Disorder-mediated recovery of magnetic order. (a) Ground state characterized by a large exchange coupling $(J)$ that leads to antiferromagnetic (AFM) order. (b) Photoexcitation at low fluences results in the formation of a small density of spin vacancies and doubly occupied states with zero net moment. Effective destruction of the moment is enabled by the small charge gap. Rapid recombination events regenerate AFM order, but recombination paths that produce parallel spin alignments are prevented by the dominant AFM exchange coupling. (c) Photoexcitation at high fluences. The formation of a large density of spin vacancies relaxes the recombination constraint, leading to a long-lived disordering of the spin system. (d) Monte Carlo magnetization dynamics in a three-dimensional spin- $\frac{1}{2}$ Ising model resulting from instantaneous and random spin flips at different degrees of excitation. If a small fraction of spins is flipped, the system recovers fast. In contrast, when many spins are flipped, inducing large disorder, long-lived change in magnetization occurs.

by considering how photoexcitation modifies magnetic order in insulating quantum materials with strong SOC. Optical excitation creates electron-hole pairs, causing changes in orbital occupancies. Due to the small charge gap in $\mathrm{Sr}_{3} \mathrm{Ir}_{2} \mathrm{O}_{7}$, it is energetically favorable to create zero-spin doubly occupied sites after photoexcitation, effectively generating spin defects in the system [Fig. 5(a)] [21]. This configuration is unstable, and at low excitation density, the excited electron can recombine with its corresponding hole via the emission of phonons. Although it is in principle possible for any electron to recombine with any available hole (leading, for example, to hole motion), the strong exchange coupling, which is only slightly smaller than the charge gap, disfavors an electron from recombining with a hole that does not result in antiparallel spin alignment. This is depicted in Figs. 5(a) and 5(b). If the electron-hole pair recombine before the surrounding spin system has time to react, magnetic order can be restored without disordering the spin system beyond the initial creation of the spin defects. This suggests a direct link between electrons rapidly relaxing back to the valence band and the fast recovery of magnetic order, as measured at low excitation fluences. We indeed observe that the magnetic recovery in such a regime and the electronic relaxation times measured above $T_{\mathrm{N}}$ are comparable, suggest-

ing electron-hole recombination in the low-density limit can restore the initial spin configuration. Notably, this process is markedly nonthermal and represents magnetic perturbations beyond linear spin-wave theory. At higher excitation fluences, a large density of spin defects is created, as depicted in Fig. 5(c). When these defects are close, the energy barrier preventing electron-hole recombination events that result in nonantiparallel spin alignments is significantly reduced. This results in a mixture of parallel and antiparallel spin alignments that eventually disorders the underlying spin network, slowing down the regeneration of long-range order.

To test the feasibility of this scenario, we examine the recovery of a three-dimensional spin- $\frac{1}{2}$ Ising model after a change in magnetic order using a Monte Carlo simulation. This model neglects the specific details of $\mathrm{Sr}_{3} \mathrm{Ir}_{2} \mathrm{O}_{7}$ but instead focuses on how order is established from various disordered states while the bath temperature is kept constant. We assume the creation of doubly occupied sites can be simplified as direct spin flips, with the flipping fraction depending on the laser fluence, but no temperature change occurs. We then count how many Monte Carlo steps are required to return to the equilibrium conditions (see the Supplemental Material for details on the Monte Carlo simulation [23] and Refs. [29-31] therein). Figure 5(d) shows that, when the spin network is only weakly perturbed, order recovers quickly. However, when the system approaches complete demagnetization, the recovery slows down dramatically, and the system remains demagnetized, despite the temperature of the simulation being significantly below $T_{\mathrm{N}}$ (see also Fig. S8 in the Supplemental Material which includes simulations which increase the lattice temperature [23]). This fast recovery at small perturbations and the generation of a long-lived state at high defect concentrations in the absence of system heating is in good agreement with our experimental observations. We emphasize that this basic model does not honor the complexity of $\mathrm{Sr}_{3} \mathrm{Ir}_{2} \mathrm{O}_{7}$. For example, the model is blind to the mechanism of perturbation beyond imposing an instantaneous change, which is something that will depend on the dominant energy scale of the system. Indeed, while charge transfer in any Mott insulator is expected to produce rapid demagnetization [32], the exact nature of the SOC and the exchange interaction are likely key factors for explaining the magnitude and speed of the switching process [33]. Notwithstanding the limitations of the model, this approach allows us to explore the initial basic response of a system to a disruption of long-range order and highlights the role played by the degeneracy of the spindisordered state in preventing a rapid recovery. This points toward a central role of disorder in determining the physics of irradiated solids [34,35] and may be best understood as glasslike systems [36]. While disorder has been largely regarded as undesirable, increasing evidence suggest it might be essential in enabling and controlling functionality in systems such as solar cells and photocatalysts and might provide paths to control the excited state [37-39].

Another key observation in our data is that the electronic coupling to the low-frequency $A_{1 g}$ mode is strongly modulated by temperature and photoexcitation and correlates with changes in magnetic order. We observe that the mode spectrum is highly temperature sensitive and redshifts with increasing fluence. Moreover, the amplitude of the mode 
increases linearly with excitation and saturates at a fluence coinciding with the full demagnetization of the sample. In contrast, the optical window modulated by the high frequency $A_{1 g}$ mode does not strongly change with temperature and fluence. We note there is no consensus in the literature on the assignment of the $A_{1 g}$ vibration in $\operatorname{Sr}_{3} \operatorname{Ir}_{2} \mathrm{O}_{7}$. However, in analogy to bilayer cuprates of the similar structure, it is likely that the mode is responsible for the degree of charge transfer between the metal and the oxygen centers within the perovskite layers. This agrees with the strong coupling of the mode to visible wavelengths, which probe the charge transfer resonance. Activation of this mode has strong implications for functionality control as, for example, in cuprates, the charge transfer is known to correlate with the superconductive state [27]. Specifically, for iridates, in our experiments, charge excitation shifts the phonon equilibrium position and quenches magnetization depending on the density of spin vacancies generated. Direct perturbation of the mode could provide a pathway to modulate both the degree of spin disorder and the exchange interaction in the system and ultimately control AFM order.

\section{CONCLUSIONS}

In summary, although optical reflectivity does not couple to magnetism directly, we have outlined a strategy to infer magnetic behavior from the wavelength-dependent optical reflectivity. Using this marker, we have shown how photoexcitation of $\mathrm{Sr}_{3} \mathrm{Ir}_{2} \mathrm{O}_{7}$ can nonthermally suppress magnetic long-range order. We have found a regime, at low fluences and low temperatures, in which we can manipulate magnetic order by up to $50 \%$ but where the system can recover within a picosecond. In contrast, at higher fluences, we measure a crossover to a regime in which the magnetization is completely suppressed but where the lattice temperature remains below $T_{\mathrm{N}}$. We argue that such a crossover emanates from the generation of photo-induced spin defects that enable a pathway for spin disordering and long-lived breaking of magnetic order without significant lattice heating. We have also identified a Raman active mode that is strongly coupled to the electronic structure of the magnetic state. The light-induced, nonthermal change of spin order opens opportunities to investigate transient phases in solids. The iridates have long been thought of as the $5 d$ analogs of cuprates, but the disruption of magnetic order through static doping did not allow us to induce superconductivity. Optical excitation and manipulation of long-lived spin order via charge or through phonon control $[40,41]$ might enable a route to access and stabilize superconductivity.

\section{ACKNOWLEDGMENTS}

This paper was funded through the European Research Council (ERC) under the European Union's Horizon 2020 Research and Innovation Programme (Grant Agreement No. 758461), by PGC2018-097027-B-I00 project funded by MCIN/AEI/10.13039/501100011033/ FEDER “A way to make Europe," the Spanish State Research Agency through the "Severo Ochoa" program for Centers of Excellence in R\&D (CEX2019-000910-S), the Fundació Cellex, and Fundació Mir-Puig, the Generalitat de Catalunya through the CERCA program. A.S.J acknowledges support from the Marie Skłodowska-Curie cofund, a PROBIST fellowship (Grant Agreement No. 754510). E.P acknowledges the support from IJC2018-037384-I funded by MCIN/AEI/10.13039/501100011033. X.L. acknowledges support from the National Natural Science Foundation of China under Grant No. 11934017. Work at Brookhaven National Laboratory was supported by the U.S. Department of Energy, Office of Science, Office of Basic Energy Sciences under Contract No. DE-SC0012704. C.W. and Y. S. acknowledge the support from the National Natural Science Foundation of China (Grant No. U2032204) and the K. C. Wong Education Foundation (GJTD-2018-01).
[1] P. A. Lee, N. Nagaosa, and X.-G. Wen, Doping a Mott insulator: physics of high-temperature superconductivity, Rev. Mod. Phys. 78, 17 (2006).

[2] D. N. Basov, R. D. Averitt, and D. Hsieh, Towards properties on demand in quantum materials, Nat. Mater. 16, 1077 (2017).

[3] B. Koopmans, G. Malinowski, F. Dalla Longa, D. Steiauf, M. Fähnle, T. Roth, M. Cinchetti, and M. Aeschlimann, Explaining the paradoxical diversity of ultrafast laser-induced demagnetization, Nat. Mater. 9, 259 (2010).

[4] S. Gerber, S.-L. Yang, D. Zhu, H. Soifer, J. A. Sobota, S. Rebec, J. J. Lee, T. Jia, B. Moritz, C. Jia, A. Gauthier, Y. Li, D. Leuenberger, Y. Zhang, L. Chaix, W. Li, H. Jang, J.-S. Lee, M. Yi, G. L. Dakovski et al., Femtosecond electron-phonon lock-in by photoemission and x-ray free-electron laser, Science 357, 71 (2017)

[5] L. Vidas, D. Schick, E. Martínez, D. Perez-Salinas, A. RamosÁlvarez, S. Cichy, S. Batlle-Porro, A. S. Johnson, K. A. Hallman, R. F. Haglund, and S. Wall, Does $\mathrm{VO}_{2}$ Host a Transient Monoclinic Metallic Phase? Phys. Rev. X 10, 031047 (2020).
[6] S. Wall, D. Wegkamp, L. Foglia, K. Appavoo, J. Nag, R. F. Haglund, J. Stähler, and M. Wolf, Ultrafast changes in lattice symmetry probed by coherent phonons, Nat. Commun. 3, 721 (2012).

[7] S. Corby, E. Pastor, Y. Dong, X. Zheng, L. Francàs, M. Sachs, S. Selim, A. Kafizas, A. A. Bakulin, and J. R. Durrant, Charge separation, band-bending, and recombination in $\mathrm{WO}_{3}$ photoanodes, J. Phys. Chem. Lett. 10, 5395 (2019).

[8] S. Wall, D. Brida, S. R. Clark, H. P. Ehrke, D. Jaksch, A. Ardavan, S. Bonora, H. Uemura, Y. Takahashi, T. Hasegawa, H. Okamoto, G. Cerullo, and A. Cavalleri, Quantum interference between charge excitation paths in a solid-state Mott insulator, Nat. Phys. 7, 114 (2010).

[9] M. P. M. Dean, Y. Cao, X. Liu, S. Wall, D. Zhu, R. Mankowsky, V. Thampy, X. M. Chen, J. G. Vale, D. Casa, J. Kim, A. H. Said, P. Juhas, R. Alonso-Mori, J. M. Glownia, A. Robert, J. Robinson, M. Sikorski, S. Song, M. Kozina et al., Ultrafast energy- and momentum-resolved dynamics of magnetic correlations in the photo-doped Mott insulator $\mathrm{Sr}_{2} \mathrm{IrO}_{4}$, Nat. Mater. 15, 601 (2016). 
[10] T. Kampfrath, A. Sell, G. Klatt, A. Pashkin, S. Mährlein, T. Dekorsy, M. Wolf, M. Fiebig, A. Leitenstorfer, and R. Huber, Coherent terahertz control of antiferromagnetic spin waves, Nat. Photonics 5, 31 (2011).

[11] V. G. Sala, S. Dal Conte, T. A. Miller, D. Viola, E. Luppi, V. Véniard, G. Cerullo, and S. Wall, Resonant optical control of the structural distortions that drive ultrafast demagnetization in $\mathrm{Cr}_{2} \mathrm{O}_{3}$, Phys. Rev. B 94, 014430 (2016).

[12] M. Först, R. I. Tobey, S. Wall, H. Bromberger, V. Khanna, A. L. Cavalieri, Y. D. Chuang, W. S. Lee, R. Moore, W. F. Schlotter, J. J. Turner, O. Krupin, M. Trigo, H. Zheng, J. F. Mitchell, S. S. Dhesi, J. P. Hill, and A. Cavalleri, Driving magnetic order in a manganite by ultrafast lattice excitation, Phys. Rev. B 84, 241104 (2011).

[13] D. Afanasiev, A. Gatilova, D. J. Groenendijk, B. A. Ivanov, M. Gibert, S. Gariglio, J. Mentink, J. Li, N. Dasari, M. Eckstein, T. Rasing, A. D. Caviglia, and A. V. Kimel, Ultrafast Spin Dynamics in Photodoped Spin-Orbit Mott Insulator $\mathrm{Sr}_{2} \mathrm{IrO}_{4}$, Phys. Rev. X 9, 021020 (2019).

[14] K. R. Beyerlein, A. S. Disa, M. Först, M. Henstridge, T. Gebert, T. Forrest, A. Fitzpatrick, C. Dominguez, J. Fowlie, M. Gibert, J.-M. Triscone, S. S. Dhesi, and A. Cavalleri, Probing photoinduced rearrangements in the $\mathrm{NdNiO}_{3}$ magnetic spiral with polarization-sensitive ultrafast resonant soft x-ray scattering, Phys. Rev. B 102, 014311 (2020).

[15] Y. D. Chuang, W. S. Lee, Y. F. Kung, A. P. Sorini, B. Moritz, R. G. Moore, L. Patthey, M. Trigo, D. H. Lu, P. S. Kirchmann, M. Yi, O. Krupin, M. Langner, Y. Zhu, S. Y. Zhou, D. A. Reis, N. Huse, J. S. Robinson, R. A. Kaindl, R. W. Schoenlein et al., Real-Time Manifestation of Strongly Coupled Spin and Charge Order Parameters in Stripe-Ordered $\mathrm{La}_{1.75} \mathrm{Sr}_{0.25} \mathrm{NiO}_{4}$ Nickelate Crystals Using Time-Resolved Resonant X-Ray Diffraction, Phys. Rev. Lett. 110, 127404 (2013).

[16] S. L. Johnson, R. A. de Souza, U. Staub, P. Beaud, E. MöhrVorobeva, G. Ingold, A. Caviezel, V. Scagnoli, W. F. Schlotter, J. J. Turner, O. Krupin, W.-S. Lee, Y.-D. Chuang, L. Patthey, R. G. Moore, D. Lu, M. Yi, P. S. Kirchmann, M. Trigo, P. Denes et al., Femtosecond Dynamics of the Collinear-to-Spiral Antiferromagnetic Phase Transition in CuO, Phys. Rev. Lett. 108, 037203 (2012).

[17] P. Liu, B. Kim, X. Q. Chen, D. D. Sarma, G. Kresse, and C. Franchini, Relativistic GW + BSE study of the optical properties of Ruddlesden-Popper iridates, Phys. Rev. Materials 2, 075003 (2018).

[18] P. Liu and C. Franchini, Advanced first-principle modeling of relativistic Ruddlesden-Popper strontium iridates, Appl. Sci. 11, 2527 (2021).

[19] J. Bertinshaw, Y. K. Kim, G. Khaliullin, and B. J. Kim, Square lattice iridates, Annu. Rev. Condens. Matter Phys. 10, 315 (2019).

[20] D. G. Mazzone, D. Meyers, Y. Cao, J. G. Vale, C. D. Dashwood, Y. Shi, A. J. A. James, N. J. Robinson, J. Lin, V. Thampy, Y. Tanaka, A. S. Johnson, H. Miao, R. Wang, T. A. Assefa, J. Kim, D. Casa, R. Mankowsky, D. Zhu, R. Alonso-Mori et al., Laser-induced transient magnons in $\mathrm{Sr}_{3} \mathrm{Ir}_{2} \mathrm{O}_{7}$ throughout the Brillouin zone, Proc. Natl. Acad. Sci. USA 118, e2103696118 (2021)

[21] J. Kim, A. H. Said, D. Casa, M. H. Upton, T. Gog, M. Daghofer, G. Jackeli, J. van den Brink, G. Khaliullin, and B. J. Kim, Large Spin-Wave Energy Gap in the Bilayer Iridate
$\mathrm{Sr}_{3} \mathrm{Ir}_{2} \mathrm{O}_{7}$ : Evidence for Enhanced Dipolar Interactions Near the Mott Metal-Insulator Transition, Phys. Rev. Lett. 109, 157402 (2012).

[22] H. Gretarsson, N. H. Sung, M. Höppner, B. J. Kim, B. Keimer, and M. Le Tacon, Two-Magnon Raman Scattering and Pseudospin-Lattice Interactions in $\mathrm{Sr}_{2} \mathrm{IrO}_{4}$ and $\mathrm{Sr}_{3} \mathrm{Ir}_{2} \mathrm{O}_{7}$, Phys. Rev. Lett. 116, 136401 (2016).

[23] See Supplemental Material at http://link.aps.org/supplemental/ 10.1103/PhysRevB.105.064409 for details of the sample, measurement setup, raw data, further analysis, and details of the Monte Carlo simulations.

[24] L. Li, P. P. Kong, T. F. Qi, C. Q. Jin, S. J. Yuan, L. E. DeLong, P. Schlottmann, and G. Cao, Tuning the $J_{\text {eff }}=\frac{1}{2}$ insulating state via electron doping and pressure in the double-layered iridate $\mathrm{Sr}_{3} \mathrm{Ir}_{2} \mathrm{O}_{7}$, Phys. Rev. B 87, 235127 (2013).

[25] H. J. Park, C. H. Sohn, D. W. Jeong, G. Cao, K. W. Kim, S. J. Moon, H. Jin, D.-Y. Cho, and T. W. Noh, Phonon-assisted optical excitation in the narrow bandgap Mott insulator $\mathrm{Sr}_{3} \mathrm{Ir}_{2} \mathrm{O}_{7}$, Phys. Rev. B 89, 155115 (2014).

[26] R. Arita, J. Kuneš, A. V. Kozhevnikov, A. G. Eguiluz, and M. Imada, $A b$ initio Studies on the Interplay between Spin-Orbit Interaction and Coulomb Correlation in $\mathrm{Sr}_{2} \mathrm{IrO}_{4}$ and $\mathrm{Ba}_{2} \mathrm{IrO}_{4}$, Phys. Rev. Lett. 108, 086403 (2012).

[27] A. Ramos-Alvarez, N. Fleischmann, L. Vidas, A. FernandezRodriguez, A. Palau, and S. Wall, Probing the lattice anharmonicity of superconducting $\mathrm{YBa}_{2} \mathrm{Cu}_{3} \mathrm{O}_{7-\delta}$, Phys. Rev. B 100, 184302 (2019).

[28] L. L. Hu, M. Yang, Y. L. Wu, Q. Wu, H. Zhao, F. Sun, W. Wang, R. He, S. L. He, H. Zhang, R. J. Huang, L. F. Li, Y. G. Shi, and J. Zhao, Strong pseudospin-lattice coupling in $\mathrm{Sr}_{3} \mathrm{Ir}_{2} \mathrm{O}_{7}$ : Coherent phonon anomaly and negative thermal expansion, Phys. Rev. B 99, 094307 (2019).

[29] H. Christiansen, S. Majumder, and W. Janke, Phase ordering kinetics of the long-range Ising model, Phys. Rev. E 99, 011301 (2019).

[30] S. Marais, V. Heine, C. Nex, and E. Salje, Phenomena due to Strain Coupling in Phase Transitions, Phys. Rev. Lett. 66, 2480 (1991).

[31] S.-H. Lee, J. S. Goh, and D. Cho, Origin of the Insulating Phase and First-Order Metal-Insulator Transition in $1 T-\mathrm{TaS}_{2}$, Phys. Rev. Lett. 122, 106404 (2019).

[32] N. Bittner, D. Golež, H. U. R. Strand, M. Eckstein, and $\mathrm{P}$. Werner, Coupled charge and spin dynamics in a photo-excited Mott insulator, Phys. Rev. B 97, 235125 (2018).

[33] J. Li, N. Dasari, and M. Eckstein, Ultrafast dynamics in relativistic Mott insulators, arXiv:2010.09253.

[34] S. Wall, S. Yang, L. Vidas, M. Chollet, J. M. Glownia, M. Kozina, T. Katayama, T. Henighan, M. Jiang, T. A. Miller, D. A. Reis, L. A. Boatner, O. Delaire, and M. Trigo, Ultrafast disordering of vanadium dimers in photoexcited $\mathrm{VO}_{2}$, Science 362, 572 (2018).

[35] D. Perez-Salinas, A. S. Johnson, D. Prabhakaran, and S. Wall, Multi-mode excitation drives disorder during the ultrafast melting of a $C_{4}$-symmetry-broken phase, Nat. Commun. 13, 238 (2022).

[36] S. Y. Zhou, M. C. Langner, Y. Zhu, Y.-D. Chuang, M. Rini, T. E. Glover, M. P. Hertlein, a G. C. Gonzalez, N. Tahir, Y. Tomioka, Y. Tokura, Z. Hussain, and R. W. Schoenlein, Glass-like recovery of antiferromagnetic spin ordering in a 
photo-excited manganite $\operatorname{Pr}_{0.7} \mathrm{Ca}_{0.3} \mathrm{MnO}_{3}$, Sci. Rep. 4, 4050 (2014).

[37] E. Pastor, J.-S. Park, L. Steier, S. Kim, M. Grätzel, J. R. Durrant, A. Walsh, and A. A. Bakulin, In situ observation of picosecond polaron self-localisation in $\alpha-\mathrm{Fe}_{2} \mathrm{O}_{3}$ photoelectrochemical cells, Nat. Commun. 10, 3962 (2019).

[38] B. Guzelturk, T. Winkler, T. W. J. Van de Goor, M. D. Smith, S. A. Bourelle, S. Feldmann, M. Trigo, S. W. Teitelbaum, H.-G. Steinrück, G. A. de la Pena, R. Alonso-Mori, D. Zhu, T. Sato, H. I. Karunadasa, M. F. Toney, F. Deschler, and A. M. Lindenberg, Visualization of dynamic polaronic strain fields in hybrid lead halide perovskites, Nat. Mater. 20, 618 (2021).
[39] A. Simonov and A. L. Goodwin, Designing disorder into crystalline materials, Nat. Rev. Chem. 4, 657 (2020).

[40] D. Fausti, R. I. Tobey, N. Dean, S. Kaiser, A. Dienst, M. C. Hoffmann, S. Pyon, T. Takayama, H. Takagi, and A. Cavalleri, Light-induced superconductivity in a stripe-ordered cuprate, Science 331, 189 (2011).

[41] R. Mankowsky, A. Subedi, M. Först, S. O. Mariager, M. Chollet, H. T. Lemke, J. S. Robinson, J. M. Glownia, M. P. Minitti, A. Frano, M. Fechner, N. A. Spaldin, T. Loew, B. Keimer, A. Georges, and A. Cavalleri, Nonlinear lattice dynamics as a basis for enhanced superconductivity in $\mathrm{YBa}_{2} \mathrm{Cu}_{3} \mathrm{O}_{6.5}$, Nature (London) 516, 71 (2014). 